\title{
Experimental and Theoretical Study on Cyanuric Chloride Derivatives as Corrosion Inhibitors for Oxyen Corrosion of Mild Steel in High Salinity Corrosive Medium
}

\author{
JINCHENG MAO*, DIFEI HAN, JINZHOU ZHAO*, XIAOJIANG YANG, CHONG LIN, \\ HENG ZHANG
}

State Key Laboratory of Oil and Gas Reservoir Geology and Exploitation, Southwest Petroleum University, Chengdu 610500, P. R. China

\begin{abstract}
Designed and synthesized two corrosion inhibitors that can resist corrosion of low-carbon steel in high-salinity corrosive media. When these compounds are used as corrosion inhibitors in high salinity corrosive media, the laboratory evaluation results clearly showed that the two corrosion inhibitors had an excellent corrosion resistance. The synthetic corrosion inhibitors have an symmetrical structure which not only has a symmetrical appearance but also has the practical significance offorming an entire surface covering layer, which avoids the general corrosion and pitting corrosion.
\end{abstract}

Keywords: high salinity, EIS, Langmuir, Molecular Dynamic simulations

\section{Introduction}

Although many studies have been carried out in the laboratory [1-3] to simulate the $\mathrm{CO}_{2}$ corrosion behavior of pipeline steel and mild steel in Sodium chloride solution in the presence of calcium ion [4]. However, only a few articles are present on the systematic study of the oxygen corrosion behavior of mild steel with different influencing factors in high salinity solution [5]. Therefore, the investigation of the oxygen corrosion of mild steel in high salinity conditions is a scope of high theoretical and practical significance. Due to the aggressiveness of high salinity and high oxygen content inhibitors are frequently used to reduce the rate of dissolution of metals. Compounds containing $\mathrm{N}, \mathrm{S}$, and $\mathrm{O}$ have been reported as effective inhibitors [6,7]. The efficiency of an organic compound as an inhibitor is mainly dependent upon its ability to get adsorbed on a metal surface, which consists of the replacement of a water molecule at a corroding interface. The adsorption of these compounds is influenced by the electronic structure of inhibiting molecules, the steric factor, aromaticity, and electron density at the donor site, the presence of functional group such as $-\mathrm{CHO},-\mathrm{N}=\mathrm{N}, \mathrm{R}-\mathrm{OH}$, etc., molecular area, and molecular weight of the inhibitor molecule $[8,9]$. Due to the presence of polar atoms $\mathrm{N}$ in the cyanuric chloride molecule, their lone pair of electrons forms a coordination bond with the empty d orbital of the mild steel. At the same time, the $\pi$ bonds in the molecule facilitate to adsorb on the surface of mild steel to convert charge distribution and interface properties. Therefore, here we produced the cyanuric chloride derivatives as an antioxidant inhibitor under high salinity conditions. There investigative effects of different influencing factors on the performance of corrosion inhibitors. Some of the cyanuric chloride corrosion inhibitors were previously reported as efficient acidification inhibitors and there is a limited literature report is present on the cyanuric chloride derivatives functioning as an antioxidant corrosion inhibitor in high salinity conditions. No research work has been developed on the application of two cyanuric chloride derivatives as corrosion inhibitor of (YFF-1) and (YFF-2) on mild steel corrosion under high salinity conditions $[10,11]$.

In recent years, there has been a combination of experiment and theory in the research of corrosion inhibitors, and quantum chemical calculations can supplement the experimental data and even predict the unknown experimental rules with confidence. At present, the research of density functional theory (DFT) method is more and more widely used in the research field of corrosion inhibitors.

*email: jcmao@swpu.cn; zhaojzh@swpu.edu.cn 
Theoretical research is the prediction of the experimental law, and the experimental data is the verification of theoretical research. Research into corrosion inhibitors has evolved to the point where reasonable accuracy predictions can be obtained from DFT calculations. It is reported that the energy of the highest occupied molecular orbital $\left(\mathrm{E}_{\mathrm{HOMO}}\right)$ is usually related to the electron donating ability of the molecule. The higher the Еномо value of the inhibitor, the greater the ease with which electrons are supplied to the unoccupied d orbitals of the metallic iron, and the higher the inhibitor efficiency of the corrosion inhibitor. Similarly, the lower the energy value of the lowest unoccupied molecular orbital (ELUMO), the easier it is to accept electrons from the surface of the metallic iron. Therefore, higher $\mathrm{E}_{\mathrm{HOMO}}$ and lower ELUMo values usually correspond to higher inhibitor efficiency. Moreover, the smaller the HOMO-LUMO energy gap value $\left(\Delta \mathrm{E}_{\mathrm{H}-\mathrm{L}}\right)$ of the corrosion inhibitor, the higher the inhibitor efficiency of the corrosion inhibitor [12].

In the present investigation two new corrosion inhibitors (YFF-1, YFF-2) have been synthesized to investigate their inhibition effect on the corrosion of mild steel in high salinity corrosion solutions. The inhibition performance is evaluated by polarization measurement, impedance measurements, weight loss, SEM. The interaction between corrosion inhibitor and mild steel interface was analyzed by molecular dynamic simulation. The correlation of the Langmuir isotherm was tested to describe the adsorption behavior of the two corrosion inhibitors and to explain the differences in adsorption behavior based on the structure of the corrosion inhibitor.

\section{Materials and methods}

\subsection{Materials and chemicals}

Two novel cyanuric chloride derivatives were designed and synthesized referring to previously reported procedure [13]. The IUPAC name and chemical structure of synthesized cyanuric chloride derivatives are presented in Table 1.

Table 1. IUPAC name, structures, and molar mass of the two synthesized novel cyanuric chloride derivatives [14]

\begin{tabular}{|c|c|c|c|}
\hline IUPAC name & Abbreviation & Molar mass $\left(\mathrm{g} / \mathbf{m o l}^{-1}\right)$ \\
\hline $\begin{array}{c}1,1^{\prime} 1 "-(1,3,5 \text {-triazine-2,4,6- } \\
\text { trivl)tries(pyridin-1-ium)chloride }\end{array}$ & YFF-1 & 420.04 \\
\hline $\begin{array}{c}2,2^{\prime}, 2^{\prime \prime \prime}, 2^{\prime \prime \prime}, 2^{\prime \prime \prime}-((1,3,5 \text {-triazine-2,4,6- } \\
\text { triyl)tries (azanetriyl) hexametric acid }\end{array}$ &
\end{tabular}

The corrosion tests were performed on mild steel specimens, whose chemical compositions are shown in Table 2 . The mild steel specimens with dimensions of $50 \mathrm{~mm} \times 10 \mathrm{~mm} \times 3 \mathrm{~mm}$ were used for weight loss measurements, and a size of $50 \mathrm{~mm} \times 10 \mathrm{~mm} \times 2 \mathrm{~mm}$ was used for the high temperature and high pressure dynamic corrosion test. $7.5 \mathrm{~cm}$ long stem with the exposed surface area of $1.0 \mathrm{~cm}^{2}$ (rest being coated with commercially available lacquer) of mild steel specimens were used to the electrochemical measurements. The content of ions in the corrosive medium is shown in Table 3. The surface pre-treatment was carried out by polishing with 600, 800, 1000 and 1200 grit emery paper, followed by washing with double distilled water and finally degreased with acetone and then dried at 
room temperature. The electrolyte solution, was prepared from the reagent grade hydrochloric acid and double distilled water. All chemicals were analytical reagent grade without further purification.

Table 2. Chemical composition (wt.\%) of tested mild steel

\begin{tabular}{|c|c|c|c|c|c|c|c|}
\hline $\mathrm{C}$ & $\mathrm{P}$ & $\mathrm{Si}$ & $\mathrm{Mn}$ & $\mathrm{Cr}$ & $\mathrm{S}$ & $\mathrm{V}$ & Mo \\
\hline 0.31 & 0.02 & 0.18 & 0.65 & 0.95 & 0.03 & 0.08 & 0.05 \\
\hline
\end{tabular}

Table 3. Chemical composition of high salinity solution

\begin{tabular}{|c|c|c|c|c|c|c|c|c|}
\hline Ion & $\mathrm{Cl}^{-}$ & $\mathrm{SO}_{4}^{2-}$ & $\mathrm{HCO}_{3}^{-}$ & $\mathrm{Na}^{+}$ & $\mathrm{Ca}^{2+}$ & $\mathrm{Mg}^{2+}$ & $\mathrm{Br}$ & $\mathrm{I}^{-}$ \\
\hline$C(\mathrm{mg} / \mathrm{L})$ & 133658 & 150 & 33.84 & 71634.37 & 11272.5 & 1161.84 & 180 & 10 \\
\hline
\end{tabular}

\subsection{Weight loss measurements}

The weight loss measurements were carried out in a glass bottle with a capacity of $500 \mathrm{~mL}$ and corrosion pattern in high temperature and high pressure reactor (PARR 4584) for surface analysis. The effect of corrosion inhibitor concentration, temperature and ion content on the performance of the corrosion inhibitor was evaluated by the weight loss measurements. After the end of the evaluation of the prescribed time, the sample was taken out and washed thoroughly with distilled water, dried, and accurately weighed using a digital balance (accuracy: $\pm 0.1 \mathrm{mg}$ ). Each measurement was repeated three times and the average was reported. The inhibition efficiency $(E \%)$, parameters of corrosion rate $\left(C_{\mathrm{R}}\right)$, and surface coverage $(\theta)$ is calculated according to the following equation.

$$
\begin{aligned}
& C_{R}=\frac{w_{0}-w}{S t} \\
& E \%=\frac{C_{R(0)}-C_{R(i)}}{C_{R(0)}} \\
& \theta=\frac{C_{R(0)}-C_{R(i)}}{C_{R(0)}}
\end{aligned}
$$

where $w_{0}$ and $w$ are the weight values of the mild before and after corrosion, respectively. $C_{R(o)}$ nd $C_{R(i)}$ are the values of corrosion rates $\left(\mathrm{mg} \mathrm{cm}^{-2} \mathrm{~h}^{-1}\right)$ of mild steel in the absence and presence of inhibitor, $S$ is the total surface area of the mild sample being tested, $t$ is the period of corrosion, $\theta$ is the surface coverage, respectively.

\subsection{Electrochemical measurements}

Electrochemical testing is the most commonly used research method for corrosion evaluation. It can analyze the corrosion of mild steel under transient conditions, and it can also describe the whole corrosion process of mild steel in corrosive medium.

The corrosion behavior of mild at different corrosion inhibitor concentrations was discussed by Tafel polarization spectroscopy. The reference electrode is a saturated calomel electrode (SCE). A carbon rod is used as an auxiliary electrode. The working electrode is the mild steel, and the preparation steps are as follows: (a)a thin disk having a cross-sectional area of $1 \mathrm{~cm}^{2}$ were cut out from the mild steel plate. (b)After electrically connecting through the insulated copper wire, the specimens were mounted in an epoxy resin. (c)They were mechanically ground down to 1200 grit abrasive SiC papers, then washed with distilled water and absolute ethanol and dried in a stream of cold air.

All electrochemical experiments were performed in Gamry electrochemical cell in which the three electrodes connected to Gamry Instrument potentiostat/galvanostat with a Gamry framework system based on ESA 400.Gamry applications include EIS 300 for EIS measurements, DC 105 software for corrosion and Echem Analyst (version 5.50) software package for data fitting. Respectively, all potentials were measured versus SCE. The sample was immersed in the tested solution for 15 min before 
the start of the experiment to reach a steady open circuit voltage $( \pm 5 \mathrm{mV})$, and the measurements were performed in the frequency range of $10^{-2}$ to $10^{5} \mathrm{~Hz}$.

\subsection{SEM measurements}

The surface morphology of mild samples affected by different factors was analyzed using a (FEI Quanta 450) scanning electron microscope, with an accelerating voltage of $20 \mathrm{kV}$ and a magnification of 150 to 1000 times. Scanning electron microscopy analysis is the most intuitive response to the corrosion morphology of the surface after corrosion. The corrosion degree of the surface after corrosion can make an accurate judgment on the sustained release performance of the corrosion inhibitor. The surface of the pattern was first cleaned with absolute ethanol before the start of the corrosion test to ensure that the organic matter on the surface of the pattern was cleaned, then dried and weighed.

\subsection{Quantum chemical calculations}

In recent years, with the continuous improvement of computer level and the gradual improvement of related theories, quantum chemical calculation has been proved to be very effective in determining the electronic structure of molecules and elucidating the reactivity of molecules. Corrosion inhibition mechanism and corrosion inhibition performance of corrosion inhibitors are often related to parameters such as electronic structure and reactivity of corrosion inhibitor molecules. Therefore, the electronic structure and reactivity of the corrosion inhibitor molecules can be studied by quantum chemical calculation and the corrosion inhibition performance of the corrosion inhibitor can be further verified theoretically.

In order to study the mechanism of the corrosion inhibitor, the corrosion inhibitor molecules were optimized horizontally by Materials Studio 8.0, and the frequency calculation was the minimum energy point at which the stagnation point found was determined. Through Quantum chemical caculations, we can get $E_{\mathrm{HOMO}} 、 E_{\mathrm{LUMO}}$ and energy gap $\Delta E$, electron affinity potential $A$, ionization energy $I$, electronegativity $\chi$, chemical hardness $\eta$, softness $\sigma$.

\subsection{Molecular dynamic simulations}

Materials Studio's Forcite module is used to simulate the interaction between inhibitors and the iron surface. The calculation model consists of two layers: the surface layer of Fe $\left(\begin{array}{lll}1 & 0 & 0\end{array}\right)$ and the water layer in which the corrosion inhibitor molecules are present. The Fe $\left(\begin{array}{lll}1 & 0 & 0\end{array}\right)$ plane thickness of the adsorption interface is 11 layers with a total of $704 \mathrm{Fe}$ atoms, and the system size is $2.30 \mathrm{~nm} \times 2.30 \mathrm{~nm} \times 1.58 \mathrm{~nm}$. The solvent layer uses an amorphous Cel I module consisting of 500 molecules of inhibitor molecules with a system size of $2.30 \mathrm{~nm} \times 2.30 \mathrm{~nm} \times 2.84 \mathrm{~nm}$. In addition, the temperature is $363 \mathrm{~K}$ integration time step is $1 \mathrm{fs}$.

\section{Results and discussions}

\subsection{Weight loss measurements}

\subsubsection{Effect of inhibitor concentration on performance}

The performance evaluation of various concentrations of different corrosion inhibitors was carried out in a glass bottle. The sample was immersed in a high salinity solution (chemical composition as shown in Table 3) in which oxygen was continuously supplied and a different concentration of corrosion inhibitor was present, and the corrosion was carried out for $72 \mathrm{~h}$ at a temperature of $363 \mathrm{~K}$. The blank test has a corrosion rate of $0.4832 \mathrm{mg} \mathrm{cm}^{-2} \mathrm{~h}^{-1}$. Experimental results are given in Figure 1. 


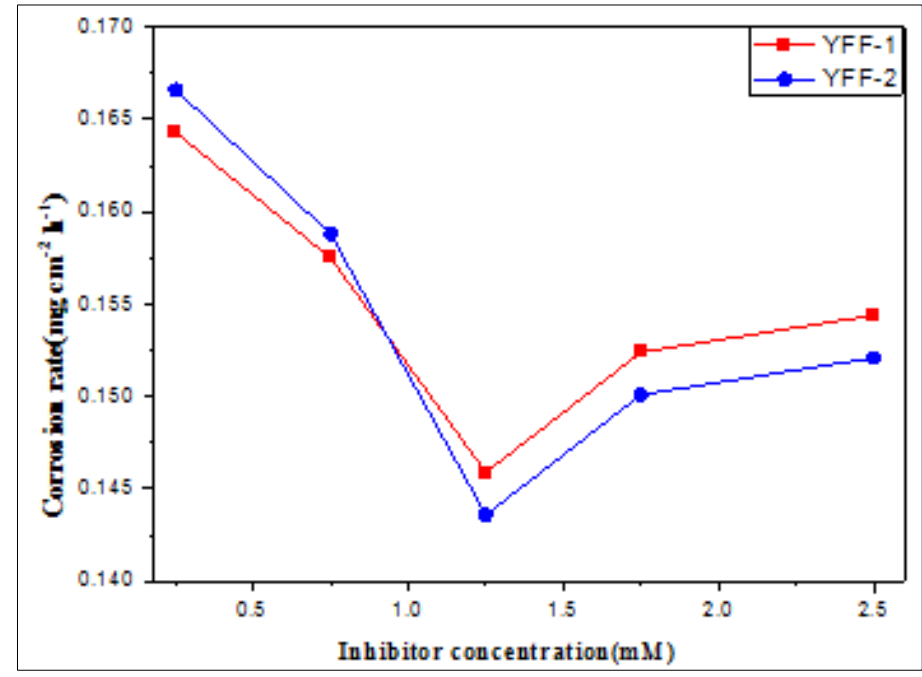

Figure 1. Corrosion rate of mild steel at different inhibitor concentrations

The experimental results show that the corrosion rate of mild steel decreases with the increase of corrosion inhibitor concentration before the concentration is less than $1.25 \mathrm{mM}$. When the concentration exceeds $1.25 \mathrm{mM}$, the corrosion rate of mild steel begins to increase, which is due to the fact that the competition between different ions in the high salinity corrosive medium significantly reduces the dispersion performance of the corrosion inhibitor and the corrosion inhibitor concentration exceeds at the optimum concentration, the anode desorption of the corrosion inhibitor occurs. At lower concentrations, the solubility of YFF-1 is better. The lone pair of electrons on the $\mathrm{N}$ can fill the empty $\mathrm{d}$ orbit of Fe to form a stable chemical adsorption with the mild steel, so the corrosion rate is lower. At high concentrations, due to the presence of carboxyl groups in the YFF-2, the solubility is better and the corrosion rate is lower. The strong polar atoms $\mathrm{O}$ and $\mathrm{N}$ in YFF-2 enhance the chemisorption, and the carboxyl groups are also complexed with $\mathrm{Fe}^{3+}$ and $\mathrm{Ca}^{2+}$ ions. The complex formed by the combination enhances the corrosion inhibition performance.

\subsubsection{Effect of temperature on performance}

The performance evaluation of various concentrations of different corrosion inhibitors was carried out in a glass bottle. The sample was immersed in a high salinity solution in the presence of $0.25 \mathrm{mM}$ and continuously supplied with oxygen, and the corrosion was carried out for $72 \mathrm{~h}$ at a temperature of 333-373 K. Experimental results are given in Figure 2.

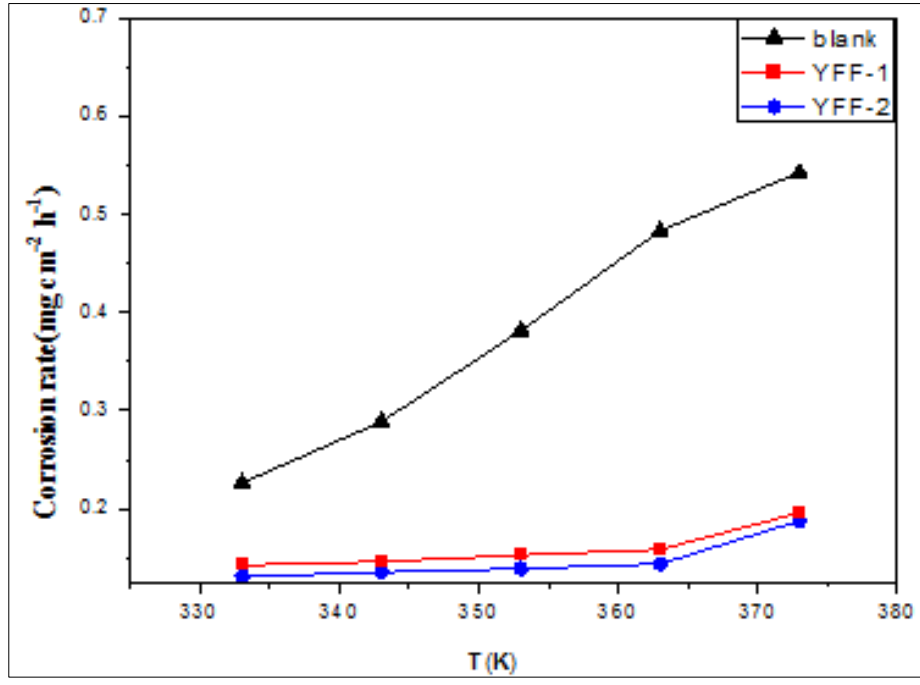

Figure 2. Corrosion rate of mild steel at different temperatures in the presence of $0.25 \mathrm{mM}$ corrosion inhibitor 
Previous literature has shown that the increase in corrosion rate with increasing temperature is due to physical adsorption. As the temperature increases, the time interval between the adsorption and desorption processes of the inhibitor molecules on the metal surface becomes shorter and shorter. Therefore, the metal surface is highly corrosive in the solution for a long time, and thus the efficiency is lowered at a high temperature.

The analysis results in Figure 2 show that under low temperature conditions, there is a synergistic effect between the corrosion inhibitor molecules, which can form a low energy stable conformation and adsorb on the surface of mild steel to prevent corrosion. As the temperature increases, the adsorption energy is higher, the adsorption is unstable, and the corrosion inhibitor is not easily oriented and adsorbed on the surface of the mild steel, resulting in an increase in corrosion rate. The adsorption performance and density of the adsorption film directly affect the corrosion process of the metal. Among the two corrosion inhibitors, the corrosion inhibition efficiency of YFF-2 is less affected by temperature due to the presence of more chemically adsorbed polar groups in the structure, and the corrosion rate is lower.

\subsubsection{Effect of $\mathrm{Ca}^{2+}$ concentration on performance}

$\mathrm{Ca}^{2+}$ easily form loose scale with anions and cause scale corrosion, which ultimately leads to an increase in corrosion rate. The effect of $\mathrm{Ca}^{2+}$ concentration on corrosion inhibition performance was carried out in a glass bottle. The sample was immersed in a high salinity solution in the presence of 0.25 $\mathrm{mM}$ and continuously supplied with oxygen, and the corrosion was carried out for $72 \mathrm{~h}$ at a temperature of $363 \mathrm{~K}$. The concentration of $\mathrm{Ca}^{2+}$ is $0-0.12 \mathrm{M}$. Experimental results are given in Figure 3.

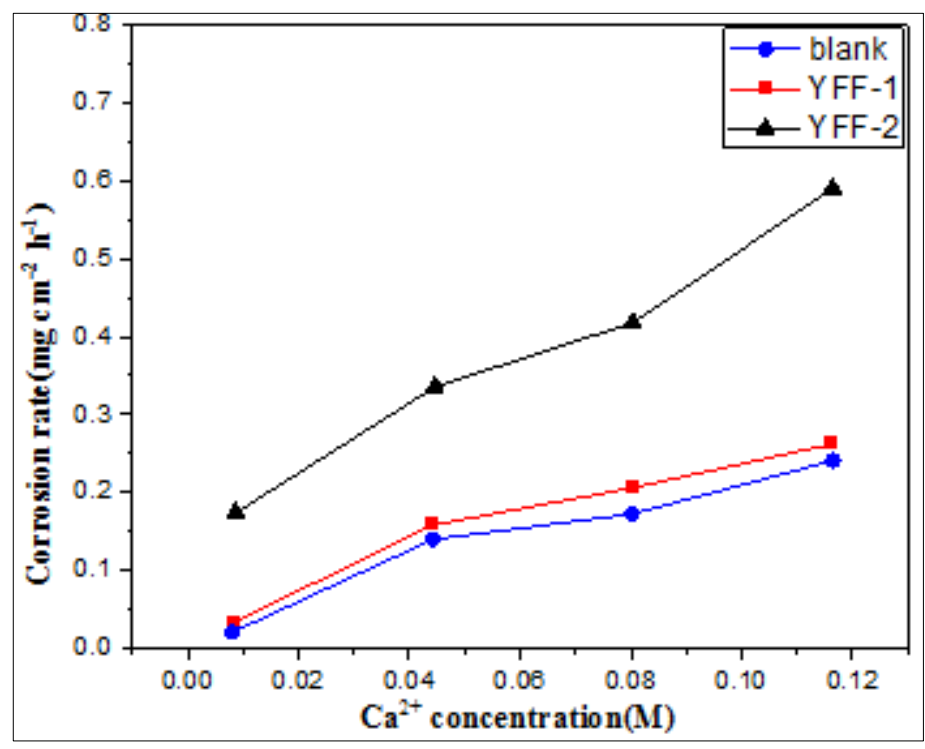

Figure 3. Corrosion rate of mild steel at different $\mathrm{Ca}^{2+}$ concentration in the presence of $0.25 \mathrm{mM}$ corrosion inhibitor

The effect of $\mathrm{Ca}^{2+}$ concentration on corrosion inhibition performance is shown in Figure 3. The experimental results show that $\mathrm{Ca}^{2+}$ have little effect on the performance of these two corrosion inhibitors. In the presence of corrosion inhibitor YFF-1, the corrosion rate of mild steel increases slowly with the increase of $\mathrm{Ca}^{2+}$ concentration, and the increase rate is gentle. The corrosion rate of YFF-2 system increases with the increase of calcium ion concentration, but the corrosion rate of mild steel is always lower than that of mild steel in the presence of YFF-1. This may be due to the carboxyl group in the YFF-2 structure, which can cooperate with $\mathrm{Ca}^{2+}$ to reduce the effect of $\mathrm{Ca}^{2+}$ on corrosion inhibition performance. 


\subsubsection{Effect of $\mathrm{Cl}^{-}$concentration on performance}

When the $\mathrm{Cl}^{-}$concentration exceeds a certain value, the very strong penetrability will damage the protective film on the metal surface, thereby causing pitting corrosion, which in turn causes local corrosion of the battery, and eventually leads to an increase in the corrosion rate. The effect of $\mathrm{Cl}^{-}$ concentration on corrosion inhibition performance was carried out in a glass bottle. The sample was immersed in a high salinity solution in the presence of $0.25 \mathrm{mM}$ corrosion inhibitor and continuously supplied with oxygen, and the corrosion was carried out for $72 \mathrm{~h}$ at a temperature of $363 \mathrm{~K}$. The concentration of $\mathrm{Cl}^{-}$is $0-4 \mathrm{M}$. Experimental results are given in Figure 4.

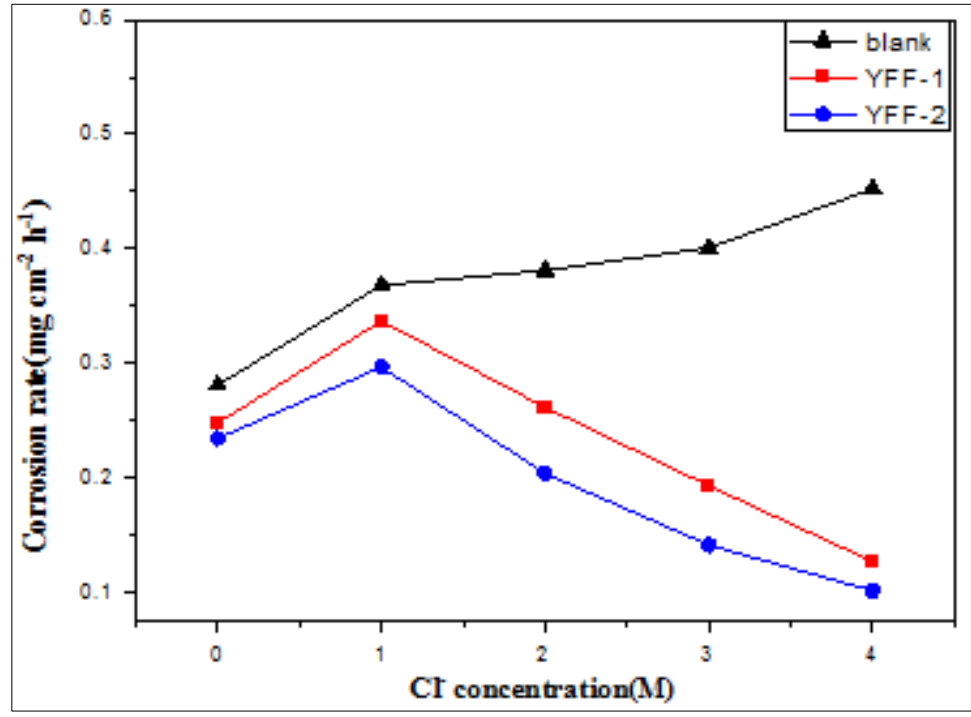

Figure 4. Corrosion rate of mild steel at different $\mathrm{Cl}^{-}$concentration

Figure 4 shows the effect of $\mathrm{Cl}^{-}$concentration on corrosion inhibition performance. When the chloride ion concentration is lower than $1 \mathrm{M}$, the corrosion rate of the mild steel in the two corrosion inhibitor systems increases, and a reddish brown corrosion product appears on the surface of the mild. As the chloride ion concentration continues to increase, the adsorption of a large amount of chloride ions on the metal surface enhances the electrostatic adsorption of the cations in the YFF-1 structure, thereby reducing the corrosion rate of the mild steel. The continuous increase in chloride ion concentration causes the ions to compete on the metal surface of the mild steel and the physical adsorption is weakened, which results in a slight increase in the corrosion rate. The corrosion rate of mild steel continues to decrease in the presence of YFF-2 because the high concentration of sodium chloride reduces the dissolved oxygen concentration. At the same time, the YFF-2 structure contains more polar groups, which can form a stable adsorption protective film on the metal surface, resulting in a decrease in the corrosion rate of the mild steel.

\subsection{Electrochemical measurements}

\subsubsection{Potentiodynamic polarization and linear polarization resistance (LPR) studies}

The results in Figure 5 show typical potential polarization curves for mild steel concentrations of corrosion inhibitors in the presence and absence of different concentrations of corrosion inhibitors. Table. 4 shows the kinetic parameters of the electrochemical corrosion, i.e. corrosion potential ( $\left.E_{\text {corr }}\right)$, cathodic and anodic Tafel slopes $\left(\beta_{a}, \beta_{c}\right)$ and corrosion current density ( $\left.I_{\text {corr }}\right)$ polarization curves obtained from the Tafel extrapolation of the polarization curves. Table 3 also includes percentage inhibition efficiency. $E \%$ is calculated according to the following equation.

$$
E(\%)=\frac{I_{c o r r}-I_{\text {corr }(i)}}{I_{\text {corr }}} \times 100
$$


where $I_{\text {corr }}$ and $I_{\text {corr (i) }}$ are corrosion current densities obtained in the absence and presence of inhibitors, respectively.

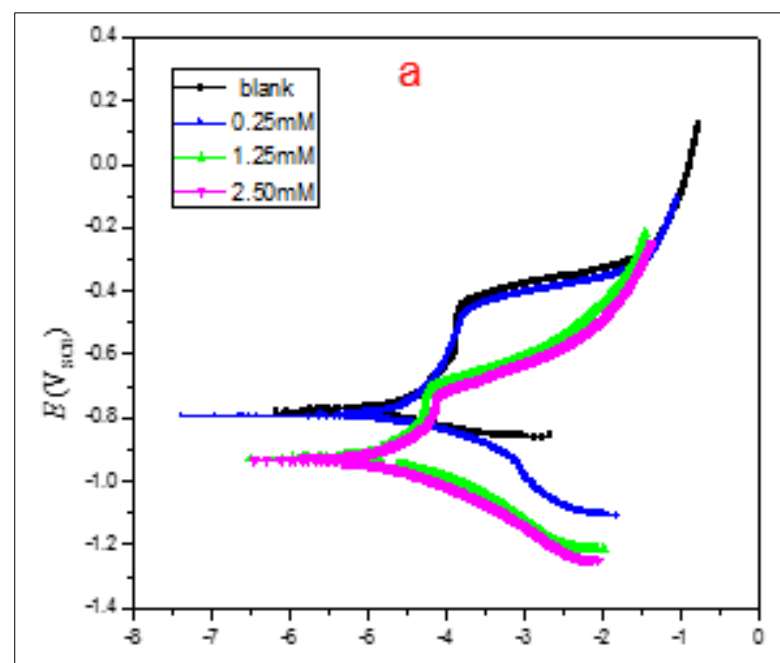

$\log i\left(\mathrm{Acm}^{2}\right)$

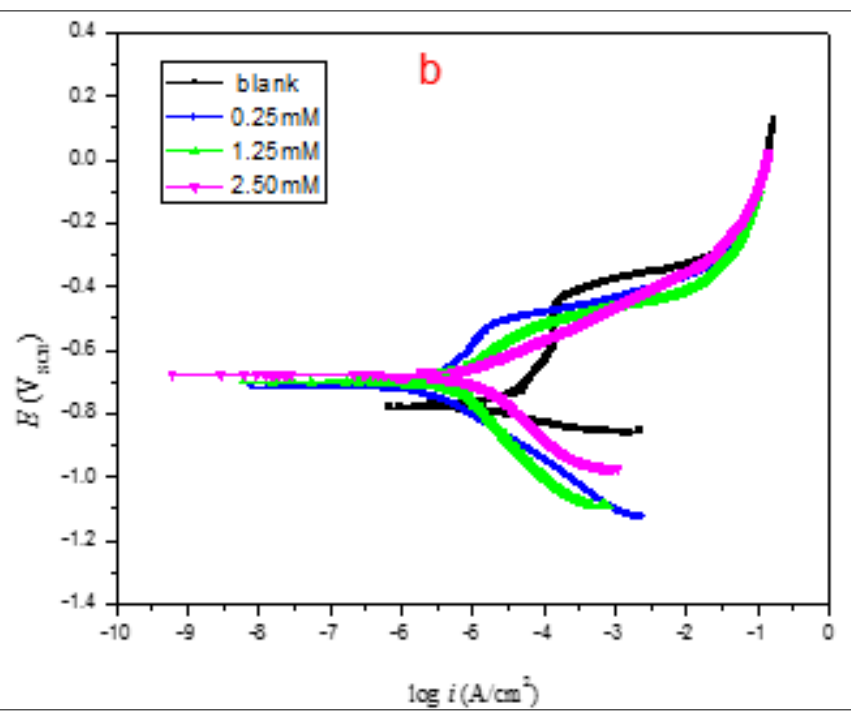

$\log i\left(\mathrm{~A} / \mathrm{cm}^{2}\right)$

Figure 5. Polarization curves for mild steel in high salinity solution at $333 \mathrm{~K}$ in the absence and presence of corrosion inhibitors: (a) YFF-1 and (b) YFF-2

Table 4. Polarization parameters for mild steel in high salinity solution at $333 \mathrm{~K}$ in the absence and presence of corrosion inhibitors

\begin{tabular}{|c|c|c|c|c|c|c|}
\hline \multirow{3}{*}{ Inhibitor } & \multirow{2}{*}{$\begin{array}{c}C \\
(\mathrm{mM})\end{array}$} & $\begin{array}{c}E_{\text {corr }} \\
(\mathrm{V} / \mathrm{SEC})\end{array}$ & $\begin{array}{c}I_{\text {corr }} \\
\left(\mathrm{mA} / \mathrm{cm}^{2}\right)\end{array}$ & $\begin{array}{c}\text { Tafel } \\
\beta_{\mathrm{a}} \\
(\mathrm{mV} / \mathrm{dec})\end{array}$ & $\begin{array}{c}\beta_{\mathrm{c}} \\
(\mathrm{mV} / \mathrm{dec})\end{array}$ & $\begin{array}{c}E \\
(\%)\end{array}$ \\
\hline \multirow{2}{*}{ Blank } & - & -0.778 & 0.0426 & 477.8 & 110.6 & - \\
\hline \multirow{3}{*}{ YFF-1 } & 0.25 & -0.793 & 0.0293 & 405.7 & 88.2 & 31.2 \\
\cline { 2 - 8 } & 1.25 & -0.929 & 0.0172 & 502.6 & 120.4 & 59.6 \\
\cline { 2 - 8 } & 2.50 & -0.932 & 0.0051 & 236.4 & 109.9 & 87.9 \\
\hline \multirow{3}{*}{ YFF-2 } & 0.25 & -0.677 & 0.0290 & 143.6 & 234.7 & 93.2 \\
\cline { 2 - 8 } & 1.25 & -0.699 & 0.0130 & 210.5 & 150.2 & 97.3 \\
\cline { 2 - 8 } & 2.50 & -0.714 & 0.0003 & 74.3 & 108.3 & 99.3 \\
\hline
\end{tabular}

The experimental results show that the current density decreases gradually after the addition of corrosion inhibitor, and the corrosion inhibition rate increases gradually. This may be due to the adsorption of YFF at the interface of mild steel/mineralization solution. It is apparent from the data in the table that the value of the corrosion potential $\left(E_{c o r r}\right)$ has a large shift. In literature, it has been reported that acompound can be classified as an anodic or a cathodic-type inhibitor when the displacement in $E_{c o r r}$ is greater than $85 \mathrm{mV}$ with reference to blank, otherwise inhibitor is treated as mixed type. In our study, the maximum displacement of $E_{c o r r}$ for the YFF-1 corrosion system is 154 $\mathrm{mV}$, the maximum displacement of the $E_{c o r r}$ system for the YFF-2 corrosion system is $101 \mathrm{mV}$, the slopes of the anode $\left(\beta_{a}\right)$ and the cathode $\left(\beta_{c}\right)$ were significantly changed in the presence of an inhibitor, indicating that neither of the two inhibitors studied was a mixed inhibitor.

The experimental results of the YFF-1 corrosion system show that as the concentration of YFF-1 increases, the $E_{c o r r}$ moves in the negative direction. This is because the $\mathrm{Cl}^{-}$in the solution adsorbs on the surface of the mild steel and causes the metal interface potential to move in the negative direction. At this time, the cation in YFF-1 is adsorbed on the surface of the mild steel by physical attraction (physical adsorption). The triazine ring and the pyridine ring contained in YFF-1 have a high electron cloud 
density and the $\mathrm{N}$ atom on the hetero ring can form a coordination bond with the d-orbital of the Fe atom. Such adsorption is a strong chemical adsorption, which increases The activation energy of the iron ionization reaction, thereby reducing the corrosion rate. The corrosion current density decreases gradually and the cathodic polarization curve changes significantly. As the concentration increases, the anode change of the polarization curve is more obvious than that of the cathode, indicating that YFF-1 is a mixed corrosion inhibitor based on the suppression of the anode.

The experimental results of YFF-2 corrosion system show that as the concentration of YFF-2 increases, the corrosion potential shifts to the positive direction, and the corrosion current density increases the corrosion inhibition efficiency. The triazine ring, N, O atom and other polar groups in YFF2 can form a strong coordination bond with the Fe atom to form a d-orbital. In addition, the carboxyl group in TYFF-2 can also be complexed with metal ions, which can form a more stable complex adsorbed on the surface of mild steel. As the concentration increases, the anode change of the polarization curve is more pronounced than that of the cathode, indicating that YFF-1 is a mixed corrosion inhibitor based on the suppression of the anode.

\subsubsection{Electrochemical impedance measurements}

Impedance measurements were made at $333 \mathrm{~K}$ in a high salinity solution in the absence and presence of a corrosion inhibitor. The Nyquist plot shows the capacitive loop (HF) range at high frequencies and the inductive loop in the low frequency (LF) range. The HF capacitor loop can be attributed to the reaction and time constant of the charge transfer bilayer surface, the homogeneity coming from the structure or interface, such as the structure or interface found during the adsorption process. The low frequency induction loop can be attributed to a relaxation process obtained by adsorbing a substance such as $\mathrm{Cl}^{-}$adsorbed adsorption on the electrode surface. It is also possible to classify the passivated surface as a low frequency. Obviously, the addition of an inhibitor results in an increase in the diameter of the semicircular capacitor (Figure $6 \mathrm{a}, 6 \mathrm{c}$ ) and the impedance of the double impedance.

Based on the shape of the Nyquist plot (Figure 6a, 6c), we decided to fit the parameters using the equivalent circuit of Figure 7. Figure 7 is an equivalent circuit diagram including solution resistance $\left(R_{s}\right)$, adsorption film resistance $\left(R_{c t}\right)$, charge transfer resistance $\left(R_{a c}\right)$, film capacitance $\left(C_{a c}\right)$, electric double layer interface capacitance $\left(C_{d l}\right)$, the above parameters can be programmed by software Get it later. The inhibition efficiencies $(E \%)$ of the tested inhibitors were calculated from the $R_{a c}$ values in the absence and presence different concentrations of corrosion inhibitors by the following equation [15]:

$$
E(\%)=\frac{R_{a c}-R_{a c}^{0}}{R_{a c}} \times 100 \%
$$

where $R_{\text {ac }}^{0}$ and $R_{\text {ac }}$ are charge transfer resistance obtained in the absence and presence of inhibitors, respectively.

Table 5. Electrochemical parameters for mild steel in high salinity solution in the absence and presence different concentrations of corrosion inhibitors at different temperatures

\begin{tabular}{|c|c|c|c|c|c|c|c|}
\hline \multirow{2}{*}{ Inhibitor } & $\begin{array}{c}\text { Concentrati } \\
\text { on }(\mathrm{mM})\end{array}$ & $\begin{array}{c}R_{s} \\
\left(\Omega \mathrm{cm}^{2}\right)\end{array}$ & $\begin{array}{c}R_{c t} \\
\left(\Omega \mathrm{cm}^{2}\right)\end{array}$ & $\begin{array}{c}R_{a c} \\
\left(\Omega \mathrm{cm}^{2}\right)\end{array}$ & $\begin{array}{c}C_{a c} \\
\left.(\mu \mathrm{F} \mathrm{cm})^{-2}\right)\end{array}$ & $\begin{array}{c}C_{d l} \\
\left.(\mu \mathrm{F} \mathrm{cm})^{-2}\right)\end{array}$ & $\begin{array}{c}E \\
(\%)\end{array}$ \\
\hline \multirow{2}{*}{ blank } & - & 2.349 & 8.169 & 6.302 & 1961 & 613.3 & - \\
\hline \multirow{3}{*}{ YFF-1 } & 0.25 & 0.662 & 9.529 & 35.36 & 290.9 & 266.8 & 85.0 \\
\cline { 2 - 10 } & 1.25 & 0.653 & 10.24 & 45.70 & 215.6 & 140.2 & 86.2 \\
\cline { 2 - 9 } & 2.50 & 0.596 & 39.15 & 61.22 & 197.2 & 93.39 & 89.7 \\
\hline \multirow{3}{*}{ YFF-2 } & 0.25 & 0.649 & 22.50 & 41.68 & 129.0 & 106.2 & 84.9 \\
\cline { 2 - 9 } & 1.25 & 0.551 & 47.84 & 62.63 & 122.4 & 80.67 & 89.9 \\
\cline { 2 - 9 } & 2.50 & 0.541 & 77.46 & 69.15 & 30.34 & 74.54 & 90.9 \\
\hline
\end{tabular}




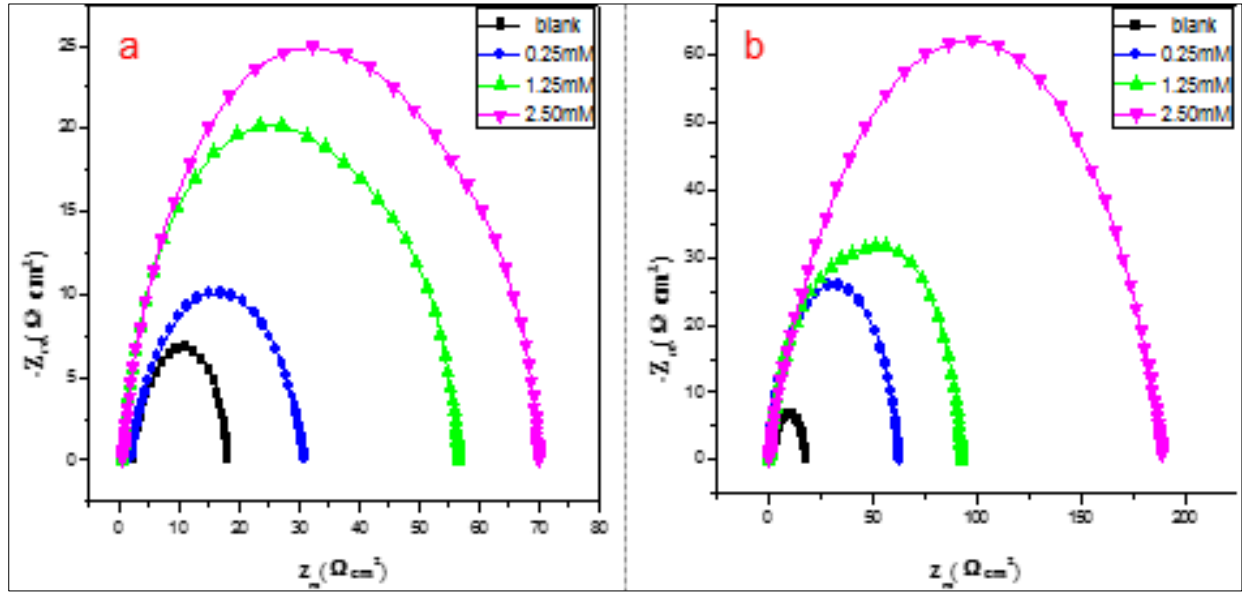

Figure 6. Nyquist plots for mild steel in high salinity solution in the absence and presence of corrosion inhibitor at 333K.a) for YFF-1, b) for YFF-2

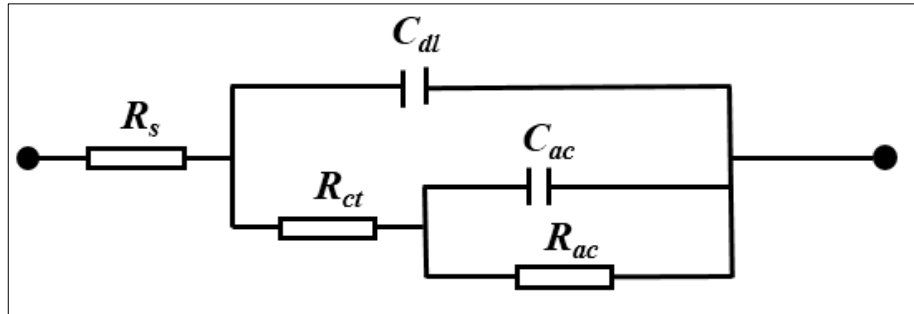

Figure 7. Equivalent circuit used to fit the obtained impedance spectra for mild steel in high salinity solution

An analysis of the data listed in Figure 6 and Table 5 shows that the Nyquist plot shows a concave capacitor loop and the radius of the capacitive arc increases as the concentration of the inhibitor increases, which means Corrosion of mild steel is hindered. The radius of the capacitive reactance arc corresponds to the charge transfer resistance $R_{a c}$, which varies significantly with the increase of the corrosion inhibitor concentration and the $R_{a c}$ value of the YFF-2 corrosion system is always greater than the YFF-1 corrosion system, which indicates that the mild in the YFF-2 corrosion system more difficult to corrode. By comparing the experimental data obtained in Table 5, it can be found that the value of the electric double layer capacitor $C_{a c}$ is significantly lower than that without the corrosion inhibitor because the corrosion inhibitor has been adsorbed on the metal surface while corroding the corrosion inhibitor. To prevent water molecules from adsorbing on the surface of the mild steel, and the dielectric constant of the water molecules is much larger than the dielectric constant of the corrosion inhibitor molecules, so it is lower after being adsorbed by the corrosion inhibitor. The capacitance of the interfacial layer composed of the mild surface is much smaller than the capacitance when it is not absorbed by the corrosion inhibitor. After the addition of the corrosion inhibitor, the $R_{a c}$ value tends to increase remarkably, indicating that not only a protective film formed of the corrosion product but also an adsorption film formed of YFF was formed in the film formation system. At the same time, an increase in the $R_{c t}$ value also indicates an increase in the coverage and compactness of the anticorrosive film.

\subsection{Adsorption isotherm studies and thermodynamic analysis}

Many references have shown that organic corrosion inhibitors usually achieve corrosion inhibition by adsorption. Basic information on the interaction between the corrosion inhibitor and the metal can be represented by an isothermal adsorption curve. The values of surface coverage $(\theta)$ and the concentration of inhibitor solution $(C)$ were tested by fitting to various isotherms like Langmuir, Temkin, Freundlich and Flory-Huggins. However, the best fit was obtained with Langmuir isotherm and the isotherm can be represented as [16-18]: 


$$
\frac{C}{\theta}=\frac{1}{K_{a d s}}+C
$$

where $K_{a d s}$ is the equilibrium constant of the adsorption process, $C$ is the concentration of inhibitor and $\theta$ is the surface coverage. All of the above parameters can be obtained from the weight loss method experiment. In addition to this, thermodynamic parameters Standard free energy ( $\Delta G_{\text {ads }}^{o}$ ), enthalpy $\left(\Delta H_{\text {ads }}^{\mathrm{o}}\right)$ and entropy $\left(\Delta S_{\text {ads }}^{\mathrm{o}}\right)$ are calculated according to the following formulas [19-21], respectively.

$$
\begin{aligned}
& \Delta G_{a d s}^{o}=-R T \ln \left(55.5 K_{a d s}\right) \\
& \ln K_{a d s}=\frac{-\Delta H_{\mathrm{ads}}^{\mathrm{o}}}{R T}+\mathrm{constant}
\end{aligned}
$$

where $R$ is the universal gas constant $8.314 \mathrm{~J} /(\mathrm{mol} \cdot \mathrm{K}), T$ is the thermodynamic temperature, and the value of 55.5 is the molar concentration of water in the solution.

$\Delta H_{\mathrm{ads}}^{\mathrm{o}}$ is calculated by linear regression between $\log K_{a d s}$ and $1000 / T$. Figure 9 shows the straight lines of $\log K_{a d s}$ versus $1000 / T$ and slope of these straight lines is equal to $\Delta H_{\text {ads }}^{\mathrm{o}} / 20303 R$. Now standard adsorption entropy $\left(\Delta S_{a d s}^{o}\right)$ is obtained by the thermodynamic basic equation [22]:

$$
\Delta S_{\mathrm{ads}}^{\mathrm{o}}=\frac{\Delta H_{\mathrm{ads}}^{\mathrm{o}}-\Delta G_{a d s}^{o}}{T}
$$

\begin{tabular}{|c|c|c|c|c|c|}
\hline Inhibitor & $\mathrm{T}(\mathrm{K})$ & $\begin{array}{c}\text { Kads } \\
\left(\mathrm{Mm}^{-1}\right)\end{array}$ & $\begin{array}{c}\Delta G_{a d s}^{o} \\
\left(\mathrm{KJ} \mathrm{mol}^{-1}\right)\end{array}$ & $\begin{array}{c}\Delta H_{a d s}^{o} \\
\left(\mathrm{KJmol}^{-1}\right)\end{array}$ & $\begin{array}{c}\Delta S_{a d s}^{o} \\
\left(\mathrm{JK}^{-1} \mathrm{~mol}^{-1}\right)\end{array}$ \\
\hline \multirow{5}{*}{ YFF-1 } & 333 & 2.35 & -2.77 & \multirow{5}{*}{-27.95} & -75.35 \\
\hline & 343 & 3.89 & -2.86 & & -72.92 \\
\hline & 353 & 5.98 & -2.94 & & -70.62 \\
\hline & 363 & 8.19 & -3.02 & & -68.43 \\
\hline & 373 & 7.06 & -3.11 & & -74.93 \\
\hline \multirow{5}{*}{ YFF-2 } & 333 & 2.91 & -1.41 & \multirow{5}{*}{-27.57} & -78.56 \\
\hline & 343 & 4.52 & -1.58 & & -75.77 \\
\hline & 353 & 6.96 & -1.75 & & -73.14 \\
\hline & 363 & 9.42 & -1.89 & & -70.74 \\
\hline & 373 & 7.54 & -1.87 & & -68.9 \\
\hline
\end{tabular}

Table 6. Thermodynamic parameters for mild steel in high salinity solution with 0.25 Mcorrosion inhibitor at different temperatures
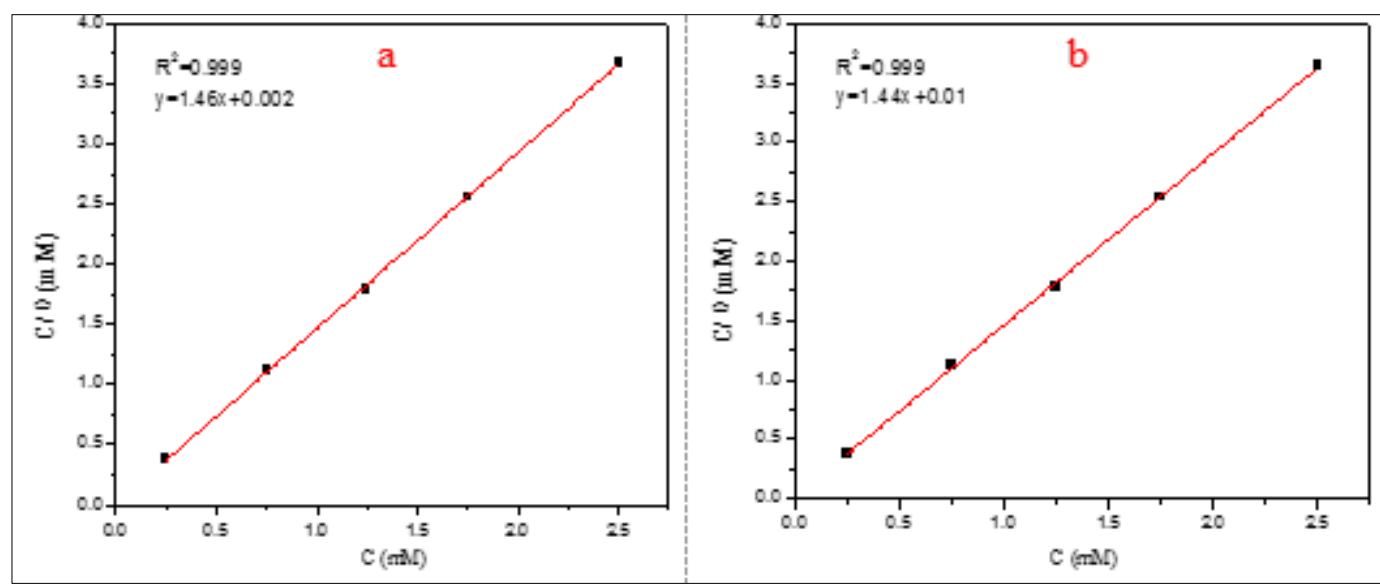

Figure 8. Langmuir adsorption plots for mild steelin high salinity solution with different concentrations at $363 \mathrm{~K}$ 


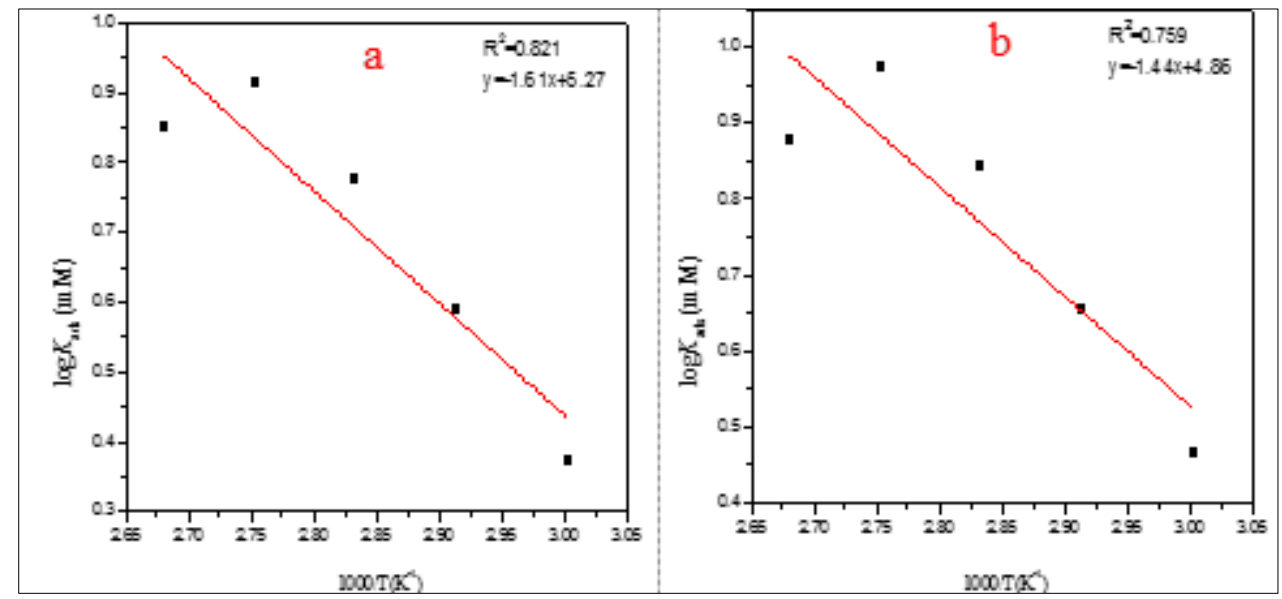

Figure 9. The relationship between $\log K_{a d s}$ and (1000/T) for mild steel in high salinity solution with different concentrations of corrosion inhibitors

Table 6 lists all calculated thermodynamic parameters. The experimental results show that the $K_{a d s}$ value gradually becomes larger as the temperature increases. The smaller the value of $\Delta G_{\text {ads }}^{o}$, the stronger the adsorption of the corrosion inhibitor on the surface of the mild steel. The value of $\Delta G_{\text {ads }}^{o}$ is $1.14 \mathrm{KJ}$ $\mathrm{mol}^{-1}$ or lower, which indicates electrostatic interaction (physical adsorption). Although those generally recognized as having a value of $\Delta G_{\text {ads }}^{o}$ of $40 \mathrm{KJ} \mathrm{mol}^{-1}$ or higher are chemical bond types (chemical adsorption). In our study, the value of $\Delta G_{\text {ads }}^{o}$ ranged from -1.41 to $3.11 \mathrm{KJ} \mathrm{mol}^{-1}$, indicating that it is possible to have both physical adsorption and chemisorption. Therefore, the value of $\Delta G_{\text {ads }}^{o}$ excludes the possibility of having only one mode of adsorption. It can also be seen from Table 6 that as the temperature increases, the $\Delta G_{\text {ads }}^{o}$ value of each corrosion inhibitor becomes more and more negative, indicating that the chemisorption is becoming more and more fragile under high temperature conditions. But in general, it shows that for the case we studied, adsorption is both physical adsorption and chemical adsorption. The negative value of $\Delta H_{\mathrm{ads}}^{\mathrm{o}}$ indicates that the adsorption of the prepared corrosion inhibitor is an exothermic process, so the corrosion inhibition performance can be affected by the temperature. As the temperature of the corrosive medium increases and is affected by the exotherm, this behavior may cause some of the corrosion inhibitor molecules to them from the surface of the mild steel.

Entropy is the driving force used to adsorb the corrosion inhibitor on the surface of the mild steel. Table 6 shows that the value of $\Delta S_{\text {ads }}^{\mathrm{o}}$ ranged from -68.4 to $-78.5 \mathrm{JK}^{-1} \mathrm{~mol}^{-1}$. The change of $\Delta S_{\mathrm{ads}}^{\mathrm{o}}$ value of corrosion inhibitor at different temperatures is not obvious, which can be explained by the fact that the corrosion inhibitor in the corrosive medium adsorbs on the surface of the mild steel and the water molecule desorption of the surface of the mild steel is dynamically balanced. In this case, the adsorption of the corrosion inhibitor is accompanied by the desorption of water molecules from the surface of the mild steel. Therefore, although the adsorption process of the inhibitor is considered to be exothermic, the desorption process of other solutes and solvents is Endothermic. The thermodynamic values obtained are the algebraic sum of organic molecular adsorption and water molecule desorption. The value of $\Delta S_{\text {ads }}^{\mathrm{o}}$ is negative and dynamically balanced because the reduction in entropy during adsorption is always greater than the increase in entropy during desorption of water molecules and is dynamically balanced.

\subsection{Surface analysis}

\subsubsection{Effect of temperature on microscopic morphology}

The effect of temperature on the micro-morphology of corrosion inhibitor effect was studied by SEM. The experiment was carried out in a high temperature and high-pressure reactor at $363 \mathrm{~K}$ for $72 \mathrm{~h}$, the partial pressure of oxygen is $0.6 \mathrm{MPa}$. The experimental results were shown in Figure 10. 


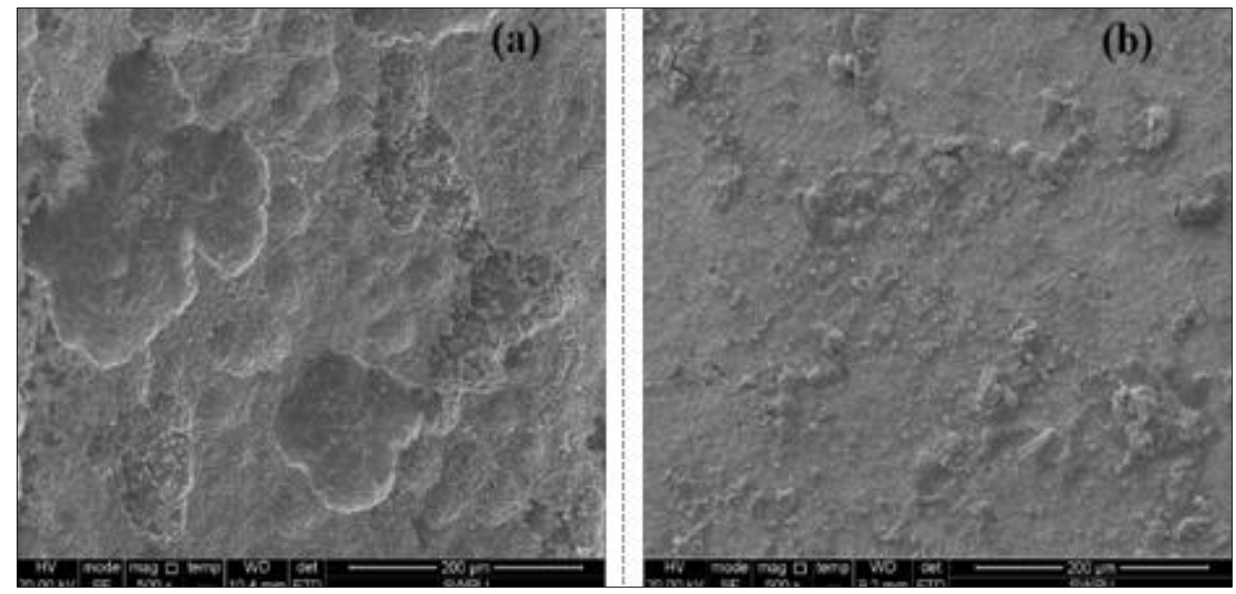

Figure 10. SEM images of mild steel surfaces:(a) without corrosion inhibitor; (b) with 1.25mM YFF-2

Figure 10(a) and Figure 10(b) shows that there is a large depressed area on the surface of the cleaned mild steel without corrosion inhibitor at $363 \mathrm{~K}$, which is the corrosion morphology after typical oxygen corrosion. The mild steel surface with corrosion inhibitor is flat without obvious corrosion depression and pitting concentration area, which indicates that the corrosion inhibitor has an excellent inhibition efficiency.

\subsubsection{Effect of $\mathrm{Ca}^{2+}$ Concentration on microscopic morphology}

The microscopic morphology analysis of the effect of Calcium ion concentration on corrosion inhibitors was studied by SEM. The experiment was performed within a high temperature and highpressure reactor at $363 \mathrm{~K}$ for $72 \mathrm{~h}$, the concentration of $\mathrm{Ca}^{2+}$ is 0 and $0.08 \mathrm{M}$. The experimental results were shown in Figure 11.

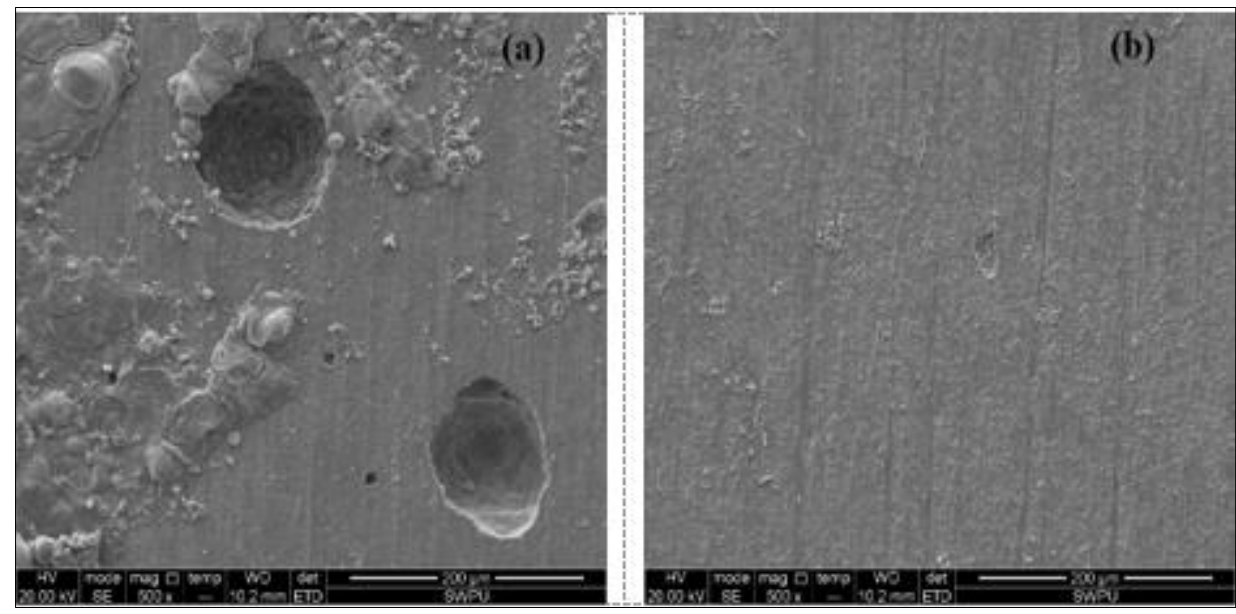

Figure 11. SEM images of mild steel surfaces in the presence of 1.25Mm YFF-2: (a)pure water;(b) $\mathrm{Ca}^{2+}(0.08 \mathrm{M})$

Figure 11 shows that large pitting and bubbling on the surface of the clean water test with corrosion inhibitor indicates severe oxygen corrosion. When the Calcium ion concentration is increased to $0.08 \mathrm{M}$, the surface of the mild steel is smooth and flat. At this time, the corrosion inhibitor has a good effect of suppressing oxygen corrosion, which also reflects the influence of calcium ion concentration on the inhibition efficiency. 


\subsubsection{Effect of $\mathrm{Cl}^{-}$Concentration on microscopic morphology}

The effect of chloride ion concentration on corrosion inhibitors was studied by SEM. The experiment was carried out with high temperature and high-pressure reactor. The sample was immersed in high salinity solution at $363 \mathrm{~K}$ for $72 \mathrm{~h}$ and the concentration of $\mathrm{Cl}^{-}$is 2 and $4 \mathrm{M}$. The experimental results were shown in Figure 12.

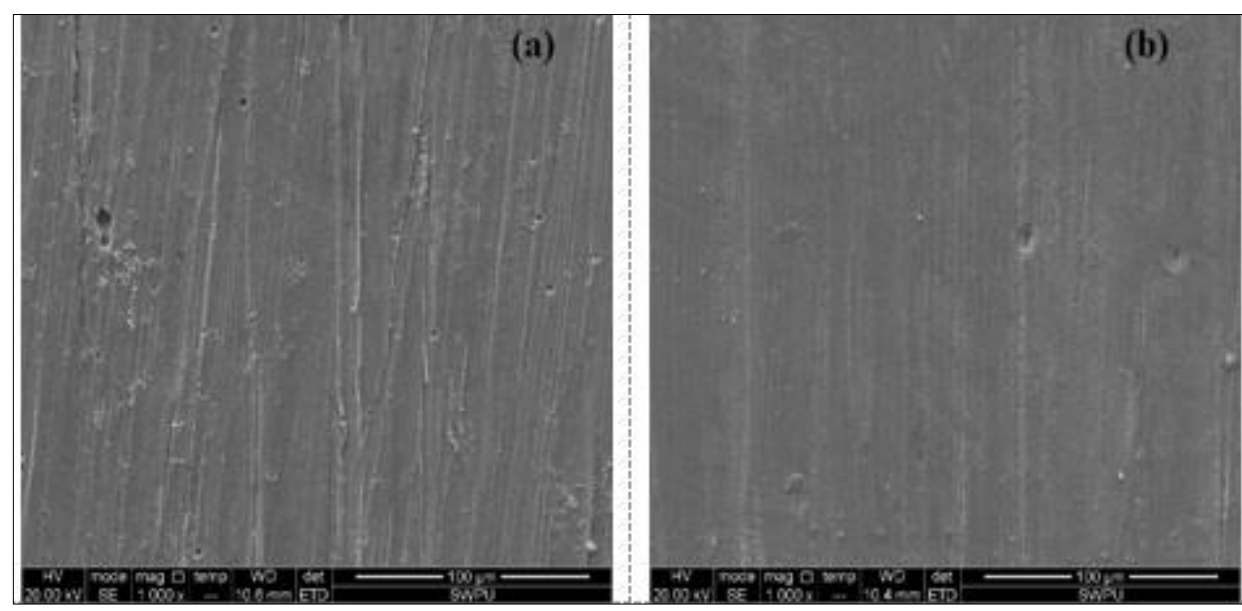

Figure 12. SEM images of mild steel surfaces in the presence of 1.25Mm YFF-2: a) $\mathrm{Cl}^{-}(2 \mathrm{M})$; b) $\mathrm{Cl}^{-}(4 \mathrm{M})$

Figure12 shows that the surface of the mild steel is flatter with the increase of the Chloride ion concentration, which indicates that the dense protective film formed on the surface of the mild steel which can effectively inhibit the occurrence of corrosion. The increase in the concentration of chloride ion in the corrosive medium reduces the amount of dissolved oxygen, so the effect of suppressing uniform corrosion is more pronounced.

\subsection{Quantum chemical calculation method}

According to the frontier orbital theory [23-26], the reactivity of molecules is related to

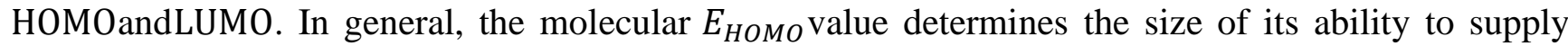
electrons outward. The level of molecular $E_{L U M O}$ represents the ability of it to accept electrons. Therefore, a higher $E_{H O M O}$ value and a lower $E_{L U M O}$ value can reflect the reactivity of a substance to some extent. For the adsorption of corrosion inhibitor molecules on the surface of metallic iron, the higher $E_{\text {номо }}$ value will make the corrosion inhibitor molecules have stronger electron supply capacity, and can form bonds with the empty d orbitals of iron iron atoms to form adsorption. The $E_{L U M O}$ value gives the corrosion inhibitor molecules a stronger electron accepting ability, which makes it easier to accept electrons from the iron surface to form an anti-bond orbital to promote adsorption. Therefore, the energy gap $\Delta E=E_{L U M O}-E_{H O M O}$ can be used to measure the size of the corrosion inhibitor molecule and iron bonding ability, the smaller the value, the stronger the bonding ability with iron and the more Easy to adsorb on metal surfaces.

According to Koopmans' theorem, under vacuum conditions, the ionization potential (I)can be approximately equal to the negative value of $E_{\text {Номо }}$.

$$
I=-E_{\text {номо }}
$$

Similarly, the electron affinity potential $(A)$ is approximately the negative of $E_{L U M O}$ :

$$
A=-E_{L U M O}
$$


Using the finite difference approximation, electronegativity $(\chi)$ and chemical hardness $(\eta)$ are as follows[27-29]:

$$
\begin{aligned}
& \chi=\frac{I+A}{2} \\
& \eta=\frac{I-A}{2}
\end{aligned}
$$

According to the Koopmans theorem, electronegativity $(\chi)$ and chemical hardness $(\eta)$ have the following relationship:

$$
\begin{aligned}
& \chi=-\frac{E_{\mathrm{HOMO}}+E_{\mathrm{LUMO}}}{2} \\
& \eta=\frac{E_{\mathrm{HOMO}}-E_{\mathrm{LUMO}}}{2}
\end{aligned}
$$

The softness $(\delta)[30]$ is the reciprocal of the chemical hardness $(\eta)$ :

$$
\delta=\frac{1}{\eta}
$$

The purpose of quantum chemical calculation is to obtain a stable geometry by optimizing the geometry of the corrosion inhibitor molecules studied, and calculate the highest occupied orbit (HOMO), the lowest empty orbit (LUMO) and its energy $\left(E_{\text {номо }}, E_{\text {LUMO }}\right)$.Energy gap $(\Delta E)$, electronegativity $(\chi)$, chemical hardness $(\eta)$, softness $(\delta)$ and other parameters to theoretically evaluate the adsorption performance of corrosion inhibitors.

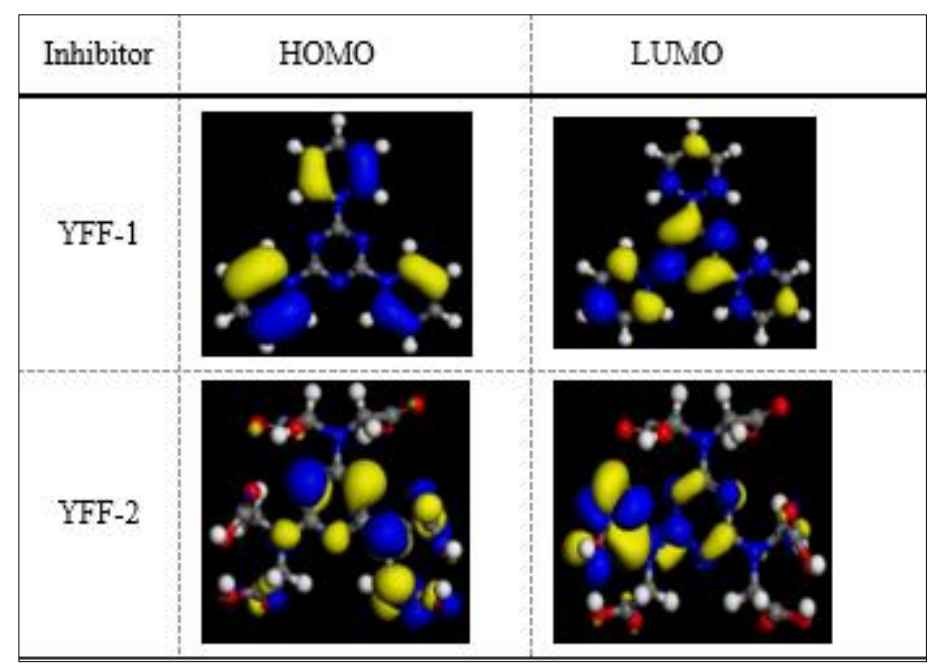

Figure 9. Structures of HOMO and LUMO of two novel cyanuric chloride derivatives

Table 7. Quantum chemical parameters for two novel cyanuric chloride derivatives

\begin{tabular}{|c|c|c|c|c|c|c|c|c|}
\hline Inhibitor & $\begin{array}{c}E_{\text {HOMO }} \\
(\mathrm{eV})\end{array}$ & $\begin{array}{c}E_{\text {LUMO }} \\
(\mathrm{eV})\end{array}$ & $I$ & $A$ & $\Delta E$ & $\eta$ & $\delta$ & $\chi$ \\
\hline YFF-1 & -2.16068 & -0.8250 & 2.1606 & 0.8250 & 1.3356 & 0.6678 & 1.4973 & 1.4928 \\
\hline YFF-2 & -6.02269 & -4.9118 & 6.0226 & 4.9118 & 1.1108 & 0.5554 & 1.8004 & 3.0113 \\
\hline
\end{tabular}

The highest occupied orbital energy $\left(E_{\text {НОмо }}\right)$ marks the electron donating ability of the inhibitor molecule. The higher the energy, the easier it is to give electrons; the lowest empty orbital energy $\left(E_{\mathrm{LUMO}}\right)$ 
indicates the ability of the inhibitor molecule to accept electrons, energy. The lower the value, the easier it is to accept electronics. It can be seen from the data in Table 7 that the corrosion inhibition rate of the corrosion inhibitor increases as the highest occupied orbital energy $\left(E_{\mathrm{HOMO}}\right)$ increases. The smaller the energy gap, the easier the electrons are excited from the highest occupied orbit, and the better the corrosion inhibition efficiency. The larger the electron affinity potential $(A)$ indicates that the molecule is more accepting electrons, and the ionization potential $(I)$ indicates that the molecule is more likely to supply electrons. Electronegativity $(\chi)$ represents the ability of a molecule to accept electrons. The greater the electronegativity, the better the corrosion inhibition performance. Softness $(\delta)$ is the reciprocal of hardness. According to the soft and hard acid-base theory, the metal atom is a soft acid, so the corrosion inhibitor of the soft base type is better.

The law of the energy gap also obeys $\Delta E$ (YFF-2) $<\Delta E$ (YFF-1), which is consistent with the data obtained from previous weight loss experiments and electrochemical experiments. ( $\left.E_{\mathrm{HOMO}}\right)$ 、 $\left(E_{\text {LUMO }}\right)$ and the energy gap $\Delta \mathrm{E}$ and other parameters can better meet the change of corrosion inhibition efficiency, and can be used to predict the corrosion inhibition efficiency of the corrosion inhibitor molecules. Because the corrosion process is a very complex process, many of the influencing factors will affect the corrosion inhibition efficiency, such as $p \mathrm{H}$, temperature, corrosion solution concentration, etc., which are not mentioned in quantum chemical calculations. So quantum chemical calculations only provide a reasonable angle for the interpretation of the corrosion inhibition mechanism. The data in Table 7 shows that I, A, $\eta, \delta$, $\chi$, etc. all have a good linear relationship with the corrosion inhibition efficiency. These parameters can also be used as evaluation parameters for corrosion inhibition efficiency.

\subsection{Molecular dynamic simulations}

Molecular dynamics simulation relies on classical Newtonian mechanics to simulate the motion state of molecules in the system. By analyzing the trajectories of molecules, the mechanics, thermodynamics and dynamics of the system are obtained. Molecular dynamics methods have become the most widely used method for calculating large, complex systems. In recent years, molecular dynamics simulation has been widely used in the field of corrosion inhibitors. It can obtain the quantitative description of the combination of corrosion inhibitor molecules and metal surface atoms, the determination of the corrosion inhibitor group, and the process of corrosion inhibitor ion adsorption film formation. And other information.

The force between the "corrosion inhibitor molecule-metal interface" of the two novel cyanuric chloride derivatives is calculated by molecular simulation to determine the corrosion inhibition performance of the corrosion inhibitor. The simulation results are shown in Figure 10.

The results of the molecular dynamic simulations in Figure 10 indicate that the two novel cyanuric chloride derivatives are almost parallel to the surface of the metal. However, from the quantum chemical calculation part, we know that the adsorption sites of two novel cyanuric chloride derivatives are not the whole molecule, but some atoms in the molecule, so the corrosion inhibitor molecules cannot be completely parallel to the metal surface. The electron donating moiety and the accepting electron moiety of the two novel cyanuric chloride derivatives are uniformly distributed throughout the molecule, and the entire molecule can provide electrons to the unoccupied d orbital of Fe to form a chemical bond and accept electrons to form an anti-bond. Therefore, the two novel cyanuric chloride derivatives are almost parallel to the metal surface, which not only helps the electrons of the corrosion inhibitor molecules to contact the metal surface, but also more effectively reduces the exposed area of the metal in the corrosive medium. In an aqueous system, the active sites of the two novel cyanuric chloride derivatives bind to the Fe $\left(\begin{array}{lll}1 & 0 & 0\end{array}\right)$ surface to immobilize the corrosion inhibitor molecules on the metal surface. It can be inferred that the corrosion inhibitor molecules can be aggregated and adsorbed on the metal surface in an almost parallel manner, thereby forming a dense adsorption film to suppress corrosion. 


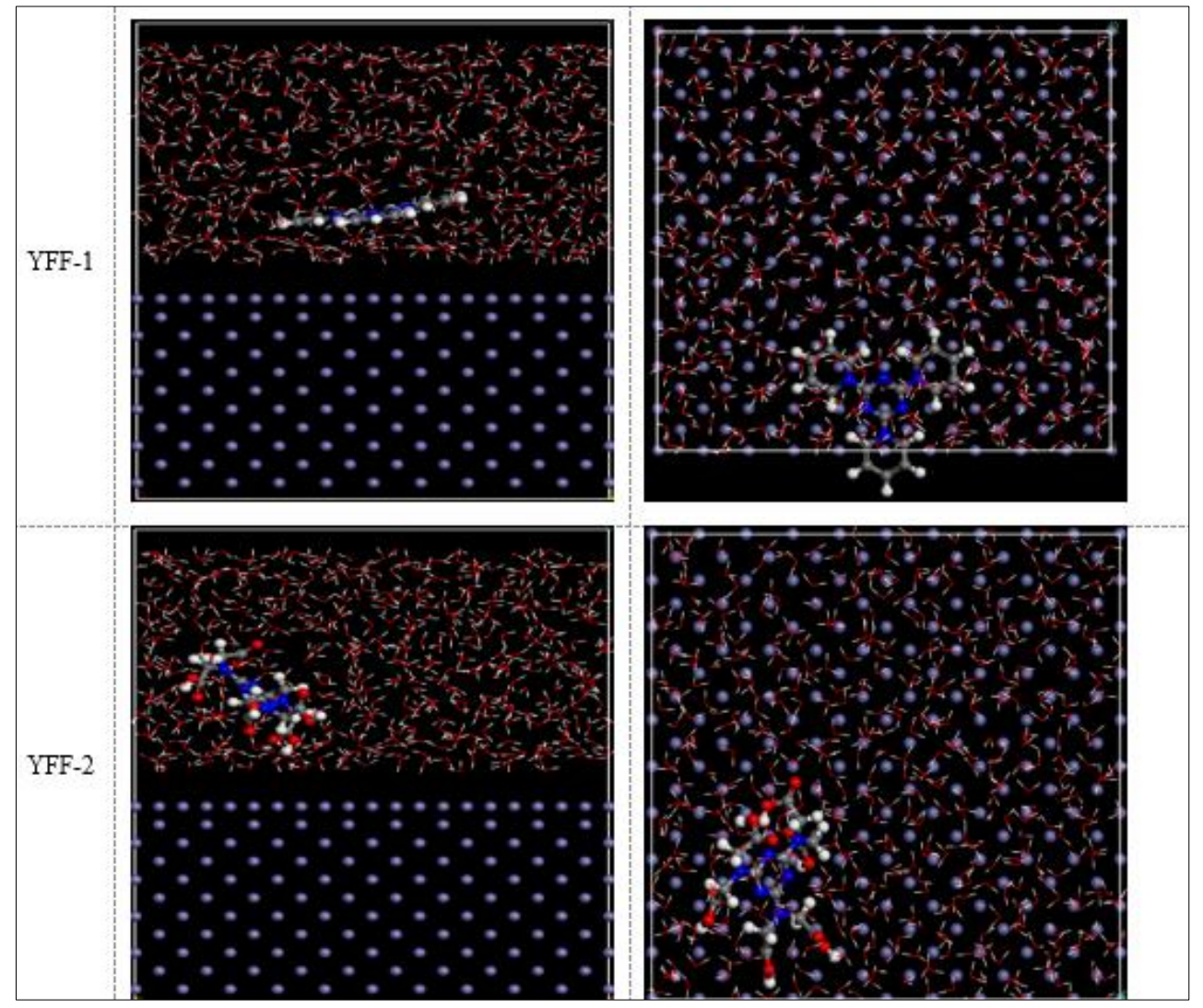

Figure 10. Top and side views of the most stable low energy configurations for the adsorption of two novel cyanuric chloride derivatives (YFF-1, YFF-2) on Fe interface obtained using molecular dynamic (MD) simulations

\section{Conclusions}

Two novel cyanuric chloride derivative corrosion inhibitors were synthesized, which have excellent dispensability in solution and good corrosion inhibition performance for mild steel in high salt solution. For two kinds of cyanuric chloride derivative corrosion inhibitors: the corrosion inhibition rate increases with the increase of the corrosion inhibitor concentration. The ions in the high concentration salinity solution have little effect on the corrosion inhibition performance, which indicates that the two corrosion inhibitors can be used as corrosion inhibitors in the high salinity solution, and the YFF-2 corrosion inhibition performance is better than YFF-1.

Polarization curve measurements show that YFF-1 and YFF-2 are mixed corrosion inhibitors that suppress cathode corrosion. The results of impedance measurements show that the corrosion of mild steel becomes more and more difficult with the increase of corrosion inhibitor concentration, and the corrosion inhibition performance of YFF-2 is better than YFF-1.

The SEM results show that the corrosion inhibitor can inhibit the occurrence of corrosion at high temperatures. The surface of the mild steel is still flat under the influence of high concentrations of calcium ions and chloride ions in the presence of corrosion inhibitors.

Acknowledgements. The research is partly supported by China Postdoctoral Science Foundation (2019 M650250), National Natural Science Foundation of China (Grant Nos. 41902303), National High Technology Research \& Development Program (2016ZX05053, 2016ZX05014-005-007) and Sichuan Province Regional Innovation Cooperation Project (2020YFQ0031). 


\section{References}

1. X. ZHANG, F. WANG, Y. HE, Y. DU, Corros Sci 43, 1417 (2001).

2. L., ZHANG, G. X., LI, W. C., F. DUY., Cheng, Journal of Materials Engineering \& Performance (2009).

3. X. YUN, L. XU, C. GAO, C. WEI, M. LU, Mater Design 36, 54 (2012).

4. S.L. WU, Z.D. CUI, G.X. ZHAO, M.L. YAN, S.L. ZHU, X.J. YANG, Appl Surf Sci $228,17$.

5. M. IOVCHEV AND I. BANKOVSKA, Desalination, 2, 221 (1982).

6. H. ASHASSI-SORKHABI, B. SHAABANI, D. SEIFZADEH, Appl Surf Sci 239, 154.

7. F. TOUHAMI, A. AOUNITI, Y. ABED, B. HAMMOUTI, K. ELKACEMI, Corros Sci 42, 929 (2000).

8. F. BENTISS, M. LAGRENEE, M. TRAISNEL, J.C. HORNEZ, Corros Sci 41, 789 (1999).

9. S.K. SHUKLA AND M.A. QURAISHi, Journal of Applied Electrochemistry, 39, 1517.

10. S.H. YOO, Y.W. KIM, K. CHUNG, N.K. KIM, J.S. KIM, Ind Eng Chem Res 52, 10880 (2013).

11. A. EL-FAHAM, K.A. DAHLOUS, Z.A.A. OTHMAN, H.A. AL-LOHEDAN, G.A. EL-MAHDY, Molecules 21, 436 (2016).

12.I. AHAMAD, R. PRASAD, M.A. QURAISHI, Corros Sci 52, 942.

13. A. EL-FAHAM, K.A. DAHLOUS, Z.A.A. OTHMAN, H.A. AL-LOHEDAN, G.A. EL-MAHDY, Molecules 21, 436 (2016).

14.X. PANG, B. HOU, L.I. WEIHUA, F. LIU, Y.U. ZHIGANG, Chinese J Chem Eng 15, 909 (2007). 15.M.A. QURAISHI, I. AHAMAD, A.K. SINGH, S.K. SHUKLA, B. LAL, V. SINGH, Materials Chemistry \& Physics 112, 1035.

16.F. BENTISS, M. TRAISNEL, M. LAGRENEE, Corros Sci 42, 127 (2000).

17. F. BENTISS, M. LAGRENEE, M. TRAISNEL, J.C. HORNEZ, Corros Sci 41, 789 (1999).

18. J. ALJOURANI, K. RAEISSI, M.A. GOLOZAR, Corros Sci 51, 1843.

19. H. ASHASSI-SORKHABI, M.R. MAJIDI, K. SEYYEDI, Appl Surf Sci 225, 176.

20. K.F. KHALED, Electrochim Acta 48, 2493 (2003).

21. S.E. NATARAJA, T.V. VENKATESHA, K. MANJUNATHA, B. POOJARY, M.K. PAVITHRA, H.C. TANDON, Corros Sci 53, 2659.

22. I. AHAMAD, R. PRASAD, M.A. QURAISHI, Corros Sci 52, 1481.

23. S. KAYA, B. TÜZÜN, C. KAYA, I.B. OBOT, J Taiwan Inst Chem E 58, 528 (2015).

24. M. LEBRINI, M. LAGRENÉE, H. VEZIN, L. GENGEMBRE, F. BENTISS, Corros Sci 47, 505.

25. N. KHALIl, Electrochim Acta 48, 2635 (2003).

26. I. AHAMAD, R. PRASAD, M.A. QURAISHI, Corros Sci 52, 1481.

27. R.G. PARR, P. Natl. Acad. Sci. Usa, 191, 277 (1995).

28. R.G. PARR, R.A. DONNELLY, M. LEVY, W.E. PALKE, J Chem Phys 68, 3801.

29. SANDERSON, R. THOMES, R.T. SANDERSON, Chemical bonds and bond energy, 1976).

30. A. HAMZI, H. ZARROK, A. ZARROUK, R. SALGHI, F. GUENOUN, Int. J. Electrochem Sc., 8, 2586 (2013)

Manuscript received: 27.07 .2020 